\title{
Integrating clinic process flow, space syntax and space adjacency analysis: Formalization of computational method in building programming
}

\author{
- Adetania Pramanik \\ Georgia Institute of Technology, USA \\ adetania.pramanik@gatech.edu \\ - John Haymaker \\ Georgia Institute of Technology, USA \\ john.haymaker@coa.gatech.edu
}

\author{
- Matthew Swarts \\ Georgia Institute of Technology, USA \\ matthew.swarts@coa.gatech.edu \\ - Craig Zimring \\ Georgia Institute of Technology, USA \\ craig.zimring@coa.gatech.edu
}

\begin{abstract}
This paper presents a computational space adjacency analysis method by integrating information and analysis from process flow methods used in the healthcare system with space syntax methods used in architecture. These methods involve similar conceptual properties related to activity, space and flow. However, their implementation in the building programming process is disconnected, and relies solely on experience and expert opinions. The basic approach for the integration was by abstracting these similar properties in the process flow diagram, space connectivity diagram, and justified plan graph into nodes and edges representations. A case study of a clinic that is currently in the construction phase was used to develop the integration procedure and comparison analysis with the actual floorplan.
\end{abstract}

Keywords: Spatial Adjacency, Process Flow, Space Syntax, Graph Theory, Building Programming

\section{Introduction}

"Garbage in, garbage out" is a familiar expression which implies how well a decision is made based on accurate data and analysis as it will determine the quality of the product output (i.e. design). In architectural design process, Pena and Parshall (2012) differentiated succinctly programming and design activities as problem seeking and problem solving, whereby a well-defined problem will lead to good problem solving. Because of the nature of problem seeking, Pena and Parshall, as well as other programing publications, offered fairly generic procedural guidance without restricting any order of the steps and methods to use. The benefit of this approach is that it allows programmers to think not only analytically but also creatively, without restrictions. However, with increasing complexity of building function, form, economy, and time, programmers face difficulties accurately defining design problems. Duerk (1993) suggested more structured, but yet generic, guidelines to develop "performance requirements" (PR) to be exchanged between programmers and designers, in order to make sure the information is analyzed appropriately and presented clearly and sufficiently at the right time (Figure 1).

Defining design problems in the form of space adjacency is commonly used because of its ability to capture various non-spatial and operational information of the occupants such as their movements, privacy and noise requirements, and many more, into space and design relationships (White, 1986). Space adjacency requirements are also frequently applied to post programming phase where various computation techniques of space planning layout are generated (Michalek, et al., 2002). Similar to building programming, a problem with implementing the space adjacency requirements lies on defining more accurate output due to the difficulty of translating various non-spatial and operational into spatial information.

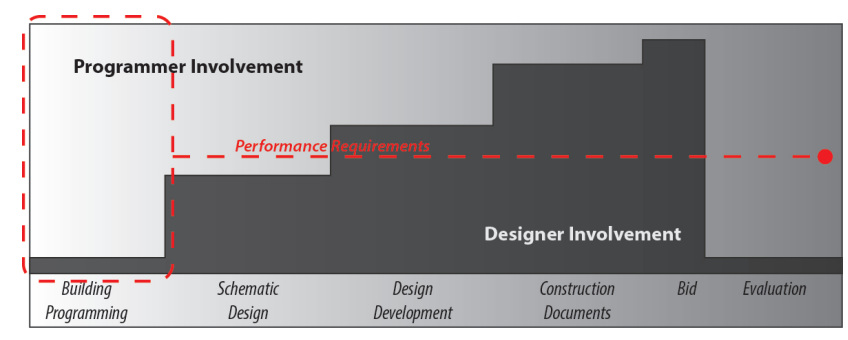

Figure 1: Programmer and Designer Involvement in the Design Process.

Healthcare buildings are arguably one of the most complicated building types (Kumar, 2011). As there are many stakeholders including patients, caregivers, clinicians, healthcare providers, and policy makers who bring various 
information and evidences for running a patient-centered outcomes process (Agency for Healthcare Research Quality, 2014). Healthcare buildings are also constrained by the process-driven activities of those stakeholders (Wurzer, 2013). Patient process flow based analysis is a common healthcare system approach to understand the complexity of various stakeholders' organizational processes. When patient process flow is successfully improved, the process of the overall operational system will also be improved (Potisek, et. al.,2007). This technique can recognize and reduce subjective variability in the system fairly quickly and easily (Haraden \& Resar, 2004). Thus, patient process flow is important non-spatial information for developing functional spaces and their relations.

Thus, this study first aims to develop computational method for space adjacency analysis by translating nonspatial information from activity flow diagrams into a space configuration analysis. Second, it aims to discover the impact of this method by analyzing similarities and differences of the proposed space adjacency analysis method with the analysis from floorplan layouts designed using current methods in industry.

\section{Process Flow}

The development of healthcare facilities in the United States has been impacted by the Affordable Care Act, which expects facilities to lower their cost of service while strengthening quality of care (medicaid.gov, 2015). In order to improve delivery, healthcare systems experts often implement patient flow analysis, where the focus is the activities of patients in the facility with little to no wait times by improving efficiency to the overall system (Potisek, et. al.,2007) (Figure 2). The goal of process flow is to recognize and eliminate variability of the structure of the delivery system such as personal and cultural preferences from the care providers (i.e., artificial variation) fairly quickly and easily (Haraden \& Resar, 2004).

Although many studies have reported the progression of technique from patient flow analysis into discrete-event or agent-based simulation in order to predict a more detailed, improved, and preferred process flow outcomes (Jacobson, et al., 2006), the challenge is how to integrate this more complex outcomes into building spaces, layout, and design. This paper implements the process flow technique for its simplest and most common form of representation of the healthcare system and because of its ability to capture and communicate overall process easily (McLaughlin \& Kaluzny, 2006).
Once the healthcare system experts achieve the desired flow efficiency, this information could be used to define the facility programming, as well as other related design problems. Patient activity flow diagram is an output diagram that is commonly developed for the facility-programming phase. Other stakeholders' activity flow diagrams could be also developed based on the goals and PRs of the project. The basic representations from the diagram are start activity, process or action step for each rounded boxes, the direction of the process flow for each line connector, decision process for each rhomboidal shape, and end activity. Connecting the activities with spaces that accommodate them seems a natural design process, yet, not many research has investigated the process of this relationship (Kim, et al., 2015). This paper explores a direct mapping approach by connecting each activity and its direction of the flow with a space ID that has space requirements to accommodate the activity and its relation to other space ID respectively (Figure 3).

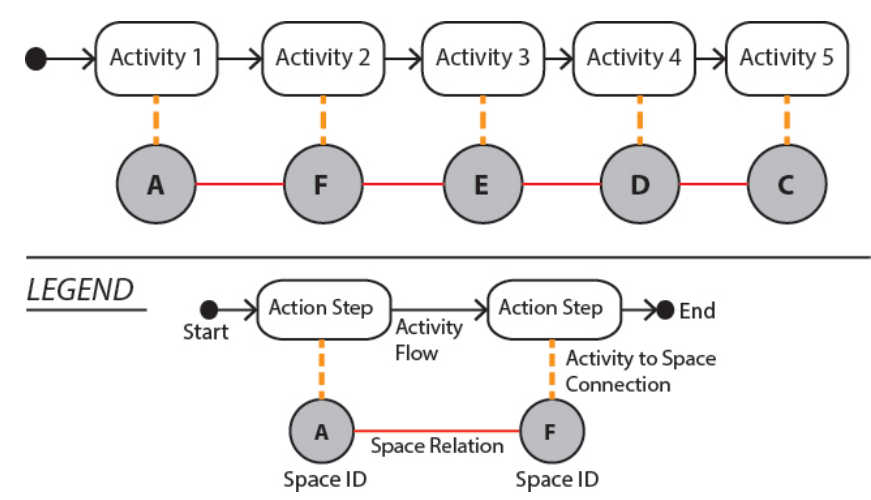

Figure 3: Direct Mapping Activity Flow to Space IDs and Space Relations.

\section{Space Adjacency}

In the field of architecture, space adjacency analysis is implemented to improve flow of movement from one space to another, related to the function of the space. In other words, the rationale of space adjacency analysis is to improve flow between spaces (White, 1986). The output of a space adjacency analysis, called an adjacency/relationship matrix, is then used as an input for space planning layout. An interesting question then arises, how are these activity flows and flow between spaces integrated? If they are integrated, there is a lack of systematic approach to integrate activity process flow into the development of space adjacency.

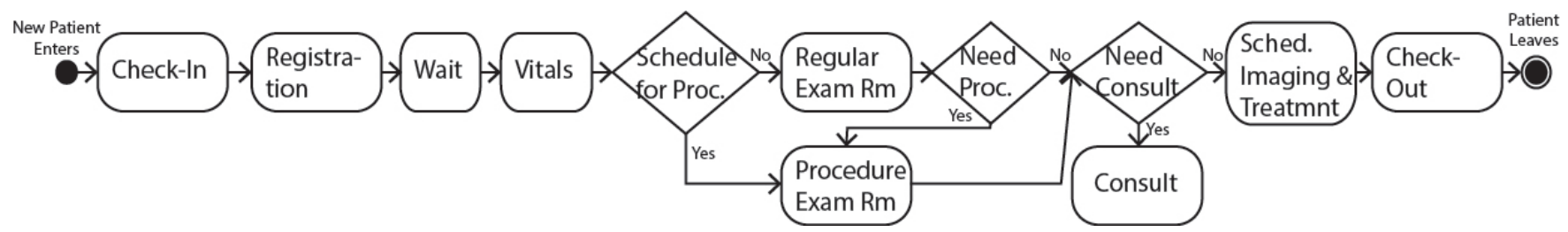

Figure 2: Activity flow diagram of patient stakeholder in the clinical case study. 
As part of building programming, any computational approach to space adjacency analysis should assist, what Pena and Parshall (2012) described as programmers' "ways of thinking", which are to name a few:

- objective thinking, where problems are defined based on facts and not influenced by subjective feelings

- comprehensive views, where all aspects of design are considered, and

- feedforward-feedback activity, where the process of defining the problems drives the design process in later phases and also allows feedback for modifying the design and improving the program.

- within a heuristic nature, which implies a pre-design process that implements incomplete or imprecise information and does not follow strict sequential steps.

The current process for analyzing adjacency requirements often relies on experience and expert opinions, which are subjective in nature. Some have developed a more systematic and less intuitive approach, for example using techniques such as travel charting and the number of journeys made (Foulds, 1983). This type of approach requires data collection from an existing facility, which would not be applicable when developing building programming for new facility.

An emerging body of early design process literature, which is mostly found on computational space planning layout (Lorenz, et al., 2015), has shown a type of objective thinking approach by utilizing quantitative properties. However, it has limited comprehensive views for not correlating the layouts with qualitative properties such as social, cultural, or organizational implications and sometimes restricting programmers into "...thoughtlessly trying out random arrangements of spaces..." (Nourian, et.al., 2013). Some of these studies rely on some form of existing adjacency matrix or initial layout (Foulds, 1983) to facilitate postprogramming or schematic design phase (refer to Figure 1). In this paper, a computational method for space adjacency analysis is explored in order to address gaps observed from the previous studies.

The common representations of the space adjacency matrix are space IDs and adjacency levels (Figure 4). Adjacency levels could be represented as qualitative degree of importance (e.g., near, neutral, and far) or as quantitative values (e.g., distance or relative distance between spaces, and number of journeys made). This paper explores a method to capture the adjacency level values from analyzing the direct mapping connections between activity process flow and space identification and relationship. Faulds (1983) offered graph theory methods as one computational approach for space planning, because of its fairly simple initial structure and ability to represent the arrangement of approximate space relationship.

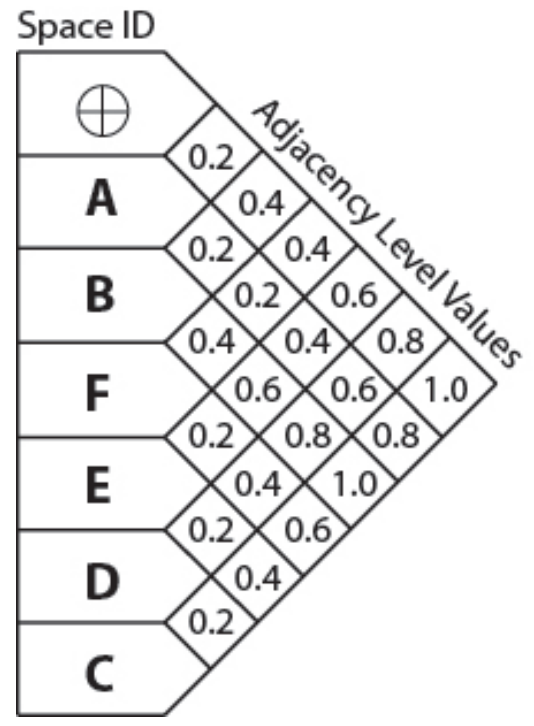

Figure 4: Representation of Space Adjacency Matrix.

\section{Space Syntax - Justified Plan Graph}

This paper explores Justified Plan Graph (JPG) theory. JPG is a graph form that has been applied in architecture as part of Space Syntax theory. This theory is interested in the correlation of space configuration with social or organizational aspects (Ostwald, 2011), which has not been the focus of the other graph theories. The basic method is to transform spaces and their access to other spaces in a floor plan layout into topological graphs as nodes and lines; abstracting out any geometrical aspects of a floor plan such as length, width, and distance. These graphs are then used to analyze connectivity and integration of spaces, as well as other measurements that explain spatial, social, or organizational characteristics.

Figure 5 summarizes an example illustrated by Ostwald (2011) for developing JPG. The process starts with a simple floor plan with doors or openings (Figure 5a) as the basis to develop a convex map representation (Figure $5 b$ ), where the defined convex spaces and each opening (connection between one space to another) are then represented as nodes and lines respectively (Figure 5c). All the nodes and connections are represented as a justified plan graph (Figure 5d). From the JPG, some configurational plan quality can be observed such as relative depth/shallowness, control/permeability, and symmetry/asymmetry (Ostwald, 2011). Example from Figure $5 \mathrm{~d}$ displays the building configuration from the entrance is five steps depths.

JPG can then be analyzed mathematically to determine each space's integration value, in which the number of spaces that are connected to each space as well as the step depth of all those connections are considered (Hillier \& Hanson, 1984) (Figure 5e). These integration values could be used to rank 
programmatic labelled spaces (Bafna, 2001). For example, the three spaces that most integrated from the figure 2 floorplan are: $\mathrm{F}$ (3.00), E (2.50), and $\mathrm{A}$ (2.50).

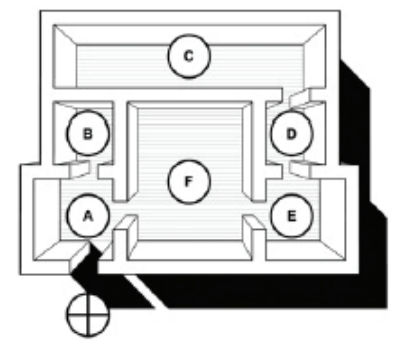

Figure 5a: Floorplan.

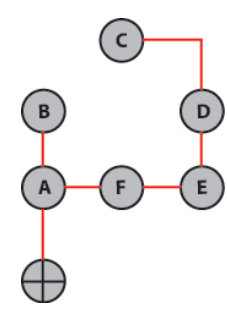

Figure 5c: Plan Graph.

$\begin{array}{ll}\text { Space } & \text { i } \\ \Theta & 1.36 \\ \text { A } & 2.50 \\ \text { B } & 1.36 \\ \text { F } & 3.00 \\ \text { E } & 2.50 \\ \text { D } & 1.66 \\ \text { C } & 1.07\end{array}$

Figure 5e: JPG Analysis.

This method of abstracting the complexity of a floor plan into configuration of spaces is similar to space adjacency analysis, where some geometrical aspects have not yet been developed in this early stage of architectural process. Thus this paper explores the possibility to utilize JPG method in the reverse order for space adjacency analysis, where the nodes and lines could be developed into convex spaces and openings, and eventually into possible floor plan layouts (Figure 6).

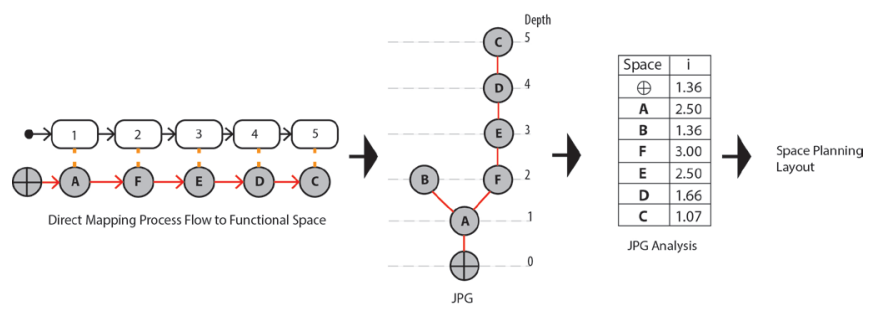

Figure 6: The Proposed Idea of Inverse JPG Method.
Space syntax theory has been used to understand environmental behavior, cognition, and evidence-based design phenomena in existing healthcare settings, such as wayfinding in hospitals, nurse movement behavior in surgical units, privacy preferences in wards, and many more (Haq \& Luo, 2012). However, only a few studies attempted to utilize space syntax theory or techniques in the architectural design process. Haq and Luo only found one study from Peponis, Zimring, and Scanlon (1996) that analyzed two alternate hospital master plans and another from Haq (2001) that reported space syntax theory as a post occupancy evaluation tool in a hospital renovation and expansion. One of the novel aims for this study is to explore the implementation of inverse JPG as part of space adjacency analysis.

\section{Methodological Procedures and Results}

The two primary research objectives are:

1. To acquire a more structured computational method for space adjacency analysis by translating non-spatial information from activity flow diagrams into a JPG analysis.

2. To validate this method by discovering similarities and differences between the output of this space adjacency method as compared with actual, designed layouts.

The support data for addressing the first research objective was collected from our observations of a clinic building programming process in industry. The study focuses on one building programming performance requirement (PR), which was: to provide the most integrated locations of assigned exam rooms and nurse station. This PR was established from previous space syntax findings that found integrated locations of these areas to be opportunity for better surveillance, social support, and subsequently better care (Haq \& Luo, 2012). The support data for addressing the second research objective was based on the floor plan from the actual project.

\section{Research Objective 1}

To address the first research objective, a UML data model was developed to describe the process observed in the industry (Figure 7) and the computational methodology of the integration of information and techniques from activity flow and space syntax into the space adjacency analysis system (Figure 8).

The process began by developing activity flows of stakeholders (patients, physicians, and nurses) with UML authoring software from the information gathered from the stakeholders and other resources (Figure 2). These flows can also be easily developed with common commercial software for healthcare system operation. We Integrated activity flows into space adjacency analysis (research objective 1a) by first identifying space IDs that accommodate the activities in the activity flows, and those that support the major spaces (Figure 9). This process assured that each activity in the process flow is 
accounted for.

Next, the connections among space IDs are assigned in accordance to the activities in the flow (Figure 10). Different configurations can be developed based on the detailed activities as well as other factors that were not captured in the activity flows. For example in configuration 2 (Figure 11), some connections were added and eliminated from configuration 1 after considering that patients could still be walking back and forth from waiting area to the reception area, and also, since the nurses would be calling the patients directly from the waiting area, access from the reception area to the vital room could be eliminated.

We integrated inverse JPG analysis into space adjacency analysis (research objective $1 \mathrm{~b}$ ) by utilizing an architectural visual programming software (e.g., Dynamo Studio). We imported the identified spaces and their connections from the UML data model, and developed a script for JPG analysis to provide the ability to adjust

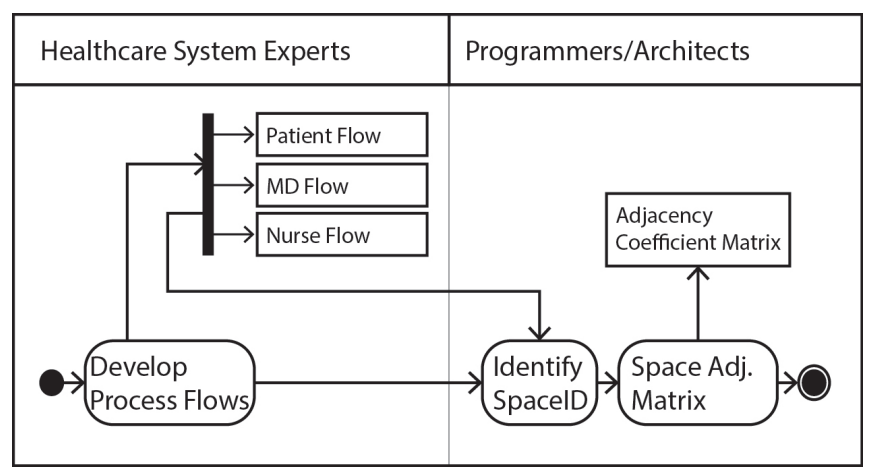

Figure 7: UML process model with stakeholders for space adjacency observed in the industry. spatial relationships until we met acceptable conditions which aligned with the building programming's objectives.

Table 1 illustrates integration values from the two configurations. The first configuration of JPG analysis resulted the three highest integration values of Nurse Station and Reception-Scheduling (11.14), and Vital Room (9.75). In the second configuration, the three highest integration values were Nurse Station (15.6), ReceptionScheduling and Exam Rooms (13.00). The three highest integration values from the second configuration met with the project PR the closest. However, it is possible that these JPG analysis and configurations would be presented to the stakeholders for determining the desired space adjacency configuration.

Once the desired space adjacency configuration was met, the matrix of depth from one space to another was exported into a .cvs file then normalized into an adjacency weight matrix (Table $2 \&$ Table 3 ).

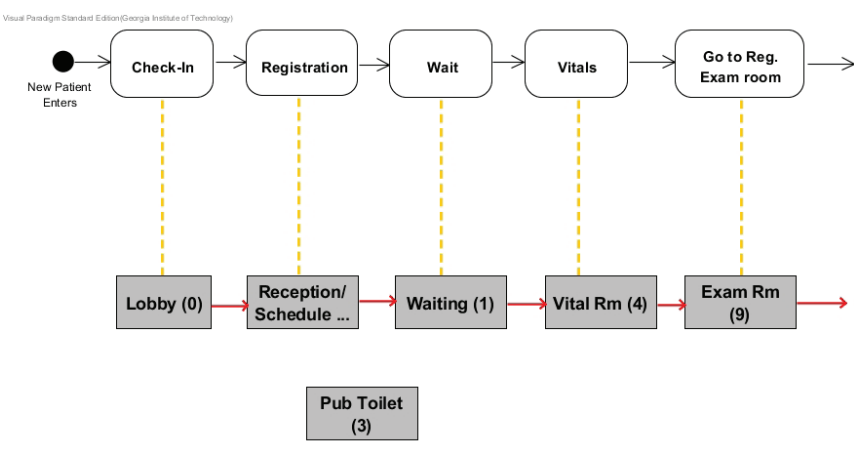

Figure 9: Identified Space IDs.

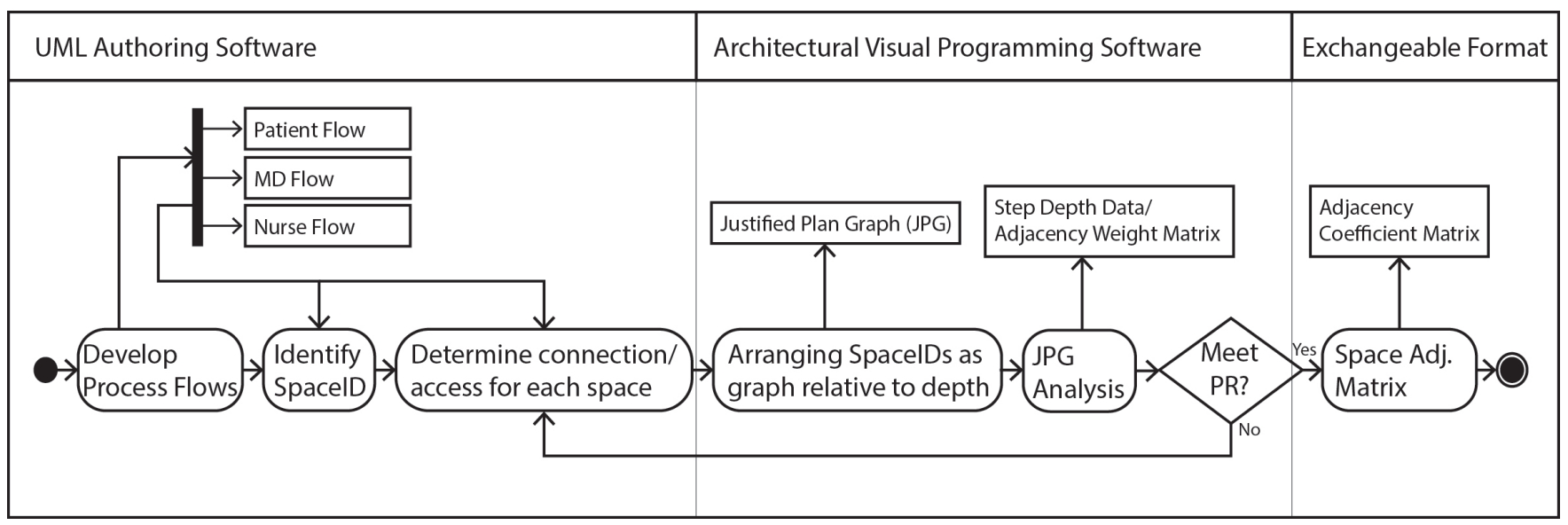

Figure 8: UML process model for computational methodology and tools of space adjacency analysis. 


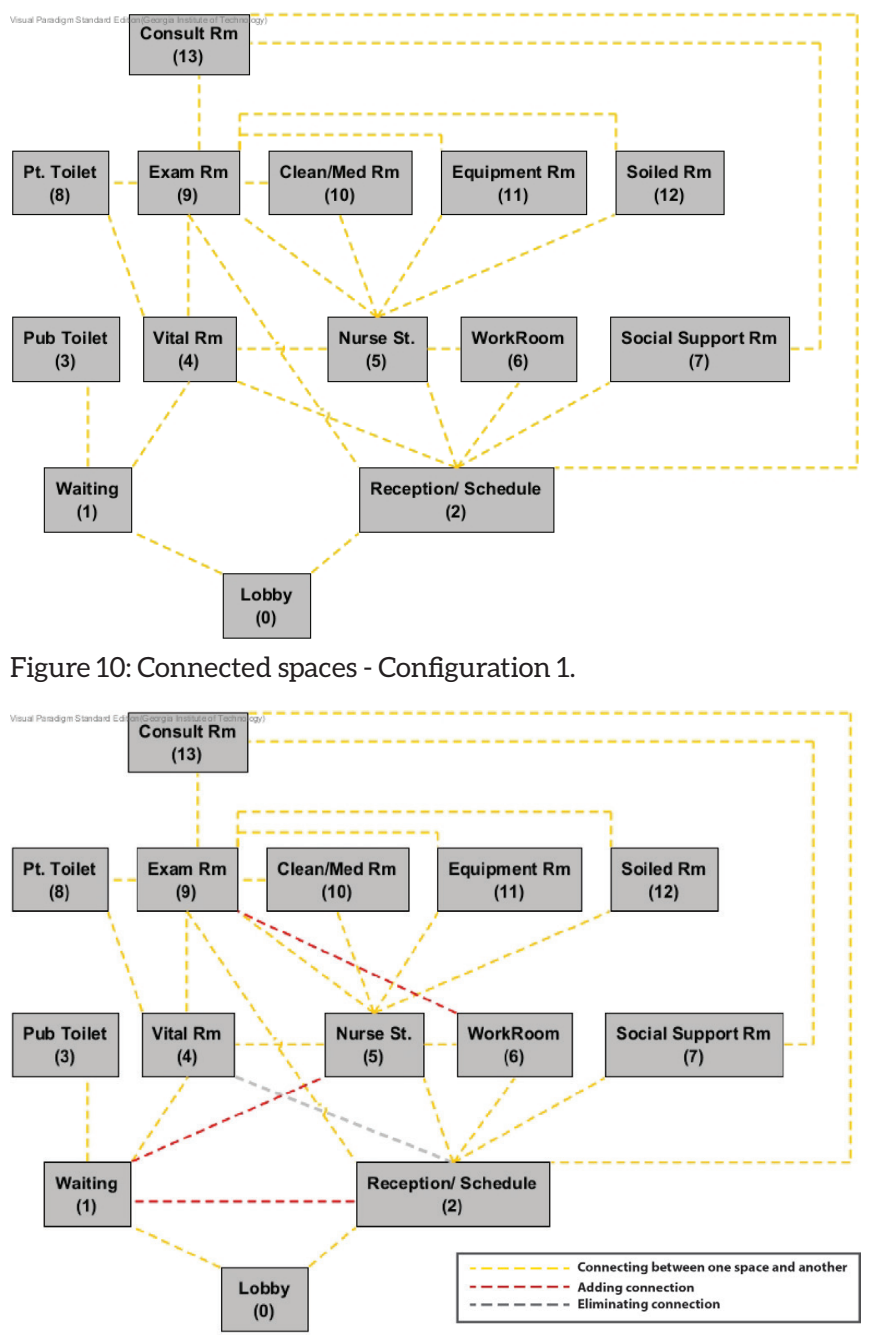

Table 1: Integration values from Configuration 1 and 2

\begin{tabular}{|c|c|c|}
\hline Space or Room & Configuration 1 & Configuration 2 \\
\hline (L) Lobby & 5.20 & 5.20 \\
\hline (W) Waiting & 4.88 & 9.75 \\
\hline (RS) Reception/Scheduling & 11.14 & 13.00 \\
\hline (Pt) Public Toilet & 2.78 & 3.00 \\
\hline (V) Vital & 9.75 & 7.80 \\
\hline (N) Nurse Station & 11.14 & 15.6 \\
\hline (Wo) Workrooms & 5.20 & 7.09 \\
\hline (Sc) Social Support Office & 4.33 & 4.59 \\
\hline (Ptt) Patient Toilet & 5.20 & 5.57 \\
\hline (Ex) Exam Rooms & 8.67 & 13.00 \\
\hline $\begin{array}{l}\text { (CE) Clean/Med \& Equipt } \\
\text { Room }\end{array}$ & 4.88 & 5.57 \\
\hline (So) Soiled Room & 4.88 & 5.57 \\
\hline (C) Consult Room & 6.00 & 6.50 \\
\hline
\end{tabular}

Figure 11: Connected spaces - Configuration 2.

Table 2: From Dynamo, 'Adjacency weight matrix' was exported into .cvs file

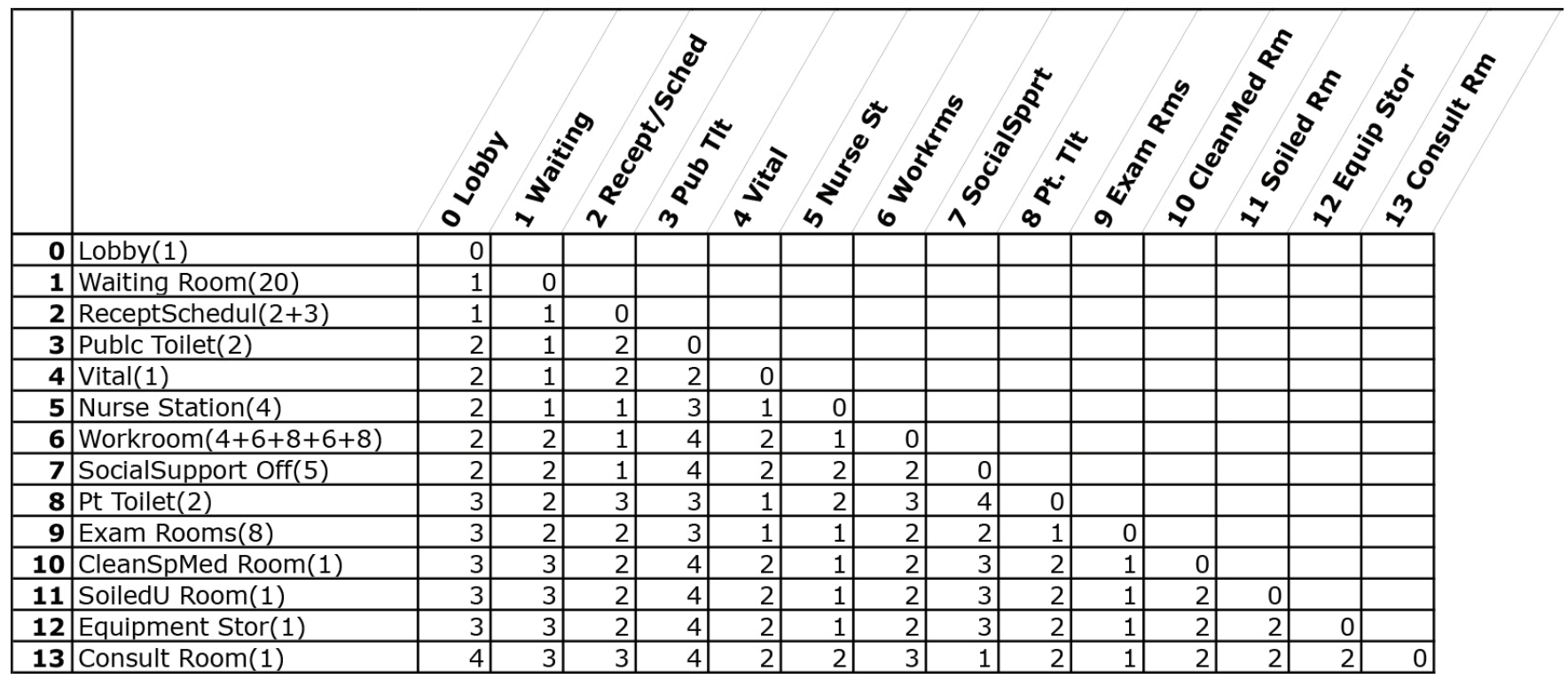




\begin{tabular}{|c|c|c|c|c|c|c|c|c|c|c|c|c|c|c|c|c|}
\hline & & & & & & & & & & & & & $y$ & $y^{2}$ & $y$ & \\
\hline & Lobby(1) & 0 & & & & & & & & & & & & & & \\
\hline 1 & Waiting Room(20) & 0.25 & 0 & & & & & & & & & & & & & \\
\hline 2 & ReceptSchedul $(2+3)$ & 0.25 & 0.25 & 0 & & & & & & & & & & & & \\
\hline 3 & Publc Toilet(2) & 0.5 & 0.25 & 0.5 & 0 & & & & & & & & & & & \\
\hline 4 & Vital(1) & 0.5 & 0.25 & 0.5 & 0.5 & 0 & & & & & & & & & & \\
\hline 5 & Nurse Station(4) & 0.5 & 0.25 & 0.25 & 0.75 & 0.25 & 0 & & & & & & & & & \\
\hline 6 & Workroom $(4+6+8+6+8)$ & 0.5 & 0.5 & 0.25 & $1 \mid$ & 0.5 & 0.25 & 0 & & & & & & & & \\
\hline 7 & SocialSupport Off(5) & 0.5 & 0.5 & 0.25 & 1 & 0.5 & 0.5 & 0.5 & 0 & & & & & & & \\
\hline 8 & Pt Toilet(2) & 0.75 & 0.5 & 0.75 & 0.75 & 0.25 & 0.5 & 0.75 & 1 & 0 & & & & & & \\
\hline 9 & Exam Rooms(8) & 0.75 & 0.5 & 0.5 & 0.75 & 0.25 & 0.25 & 0.5 & 0.5 & 0.25 & 0 & & & & & \\
\hline 10 & CleanSpMed Room(1) & 0.75 & 0.75 & 0.5 & 1 & 0.5 & 0.25 & 0.5 & 0.75 & 0.5 & 0.25 & 0 & & & & \\
\hline 11 & SoiledU Room(1) & 0.75 & 0.75 & 0.5 & 1 & 0.5 & 0.25 & 0.5 & 0.75 & 0.5 & 0.25 & 0.5 & 0 & & & \\
\hline 12 & Equipment Stor(1) & 0.75 & 0.75 & 0.5 & 1 & 0.5 & 0.25 & 0.5 & 0.75 & 0.5 & 0.25 & 0.5 & 0.5 & 0 & & \\
\hline 13 & Consult Room(1) & 1 & 0.75 & 0.75 & 1 & 0.5 & 0.5 & 0.75 & 0.25 & 0.5 & 0.25 & 0.5 & 0.5 & 0.5 & & 0 \\
\hline
\end{tabular}

This process supports characteristics of programmers' "ways of thinking" (Pena \& Parshall, 2012) by limiting programmers' subjective judgments (i.e., objective thinking) in a more structured informational linkage between healthcare system and architecture (i.e., comprehensive views). The basic approach for theseintegrationswas abstracting the complexity of information among process flow, space syntax, and space adjacency fields into nodes and edges as representations for functional spaces and their connections (heuristic nature). The output of the process provides exchangeable information between programmers and designers for the following phases of design process (feedforward activity).

\section{Research Objective 2}

To address the second research objective, the desired JPG and JPG analysis that were developed from the process flows were compared with JPG and JPG analysis from the Construction Document (CD) floorplan that were recently used in the construction phase (Figure 12).

One recognizable difference is that the $\mathrm{CD}$ floorplan presented circulation plan that had not been developed in the process flow - space adjacency phase. JPG and JPG analysis for both conditions were color coded in order to distinct the circular (green) from functional (pink) spaces (Figure 13, Figure 14, and Table 4).
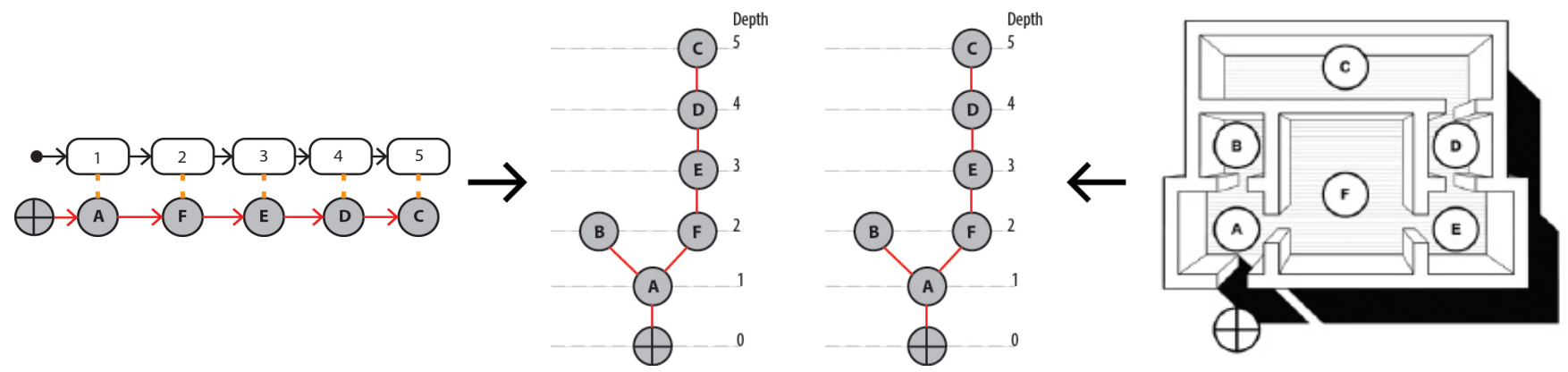

Figure 12: Comparison JPG \& JPG analysis between process flow and CD floorplan. 


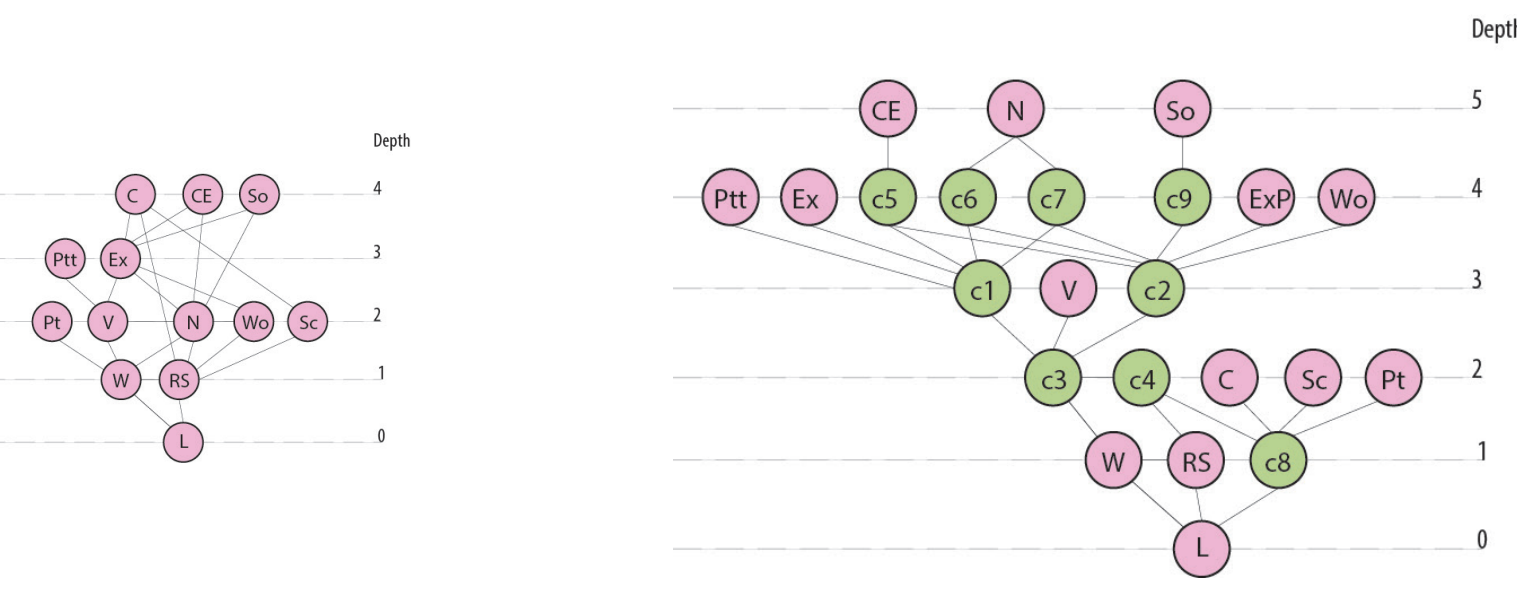

Figure 13: JPG from Process Flow.

Figure 14: JPG from Construction Document Floorplan.

Table 4: Integration values from process flow method and CD Floorplan

\begin{tabular}{|l|r|}
\hline Space from Process Flow & Integration \\
\hline (N) Nurse Station & 15.6 \\
\hline (Ex) Exam Rooms & 13.00 \\
\hline (RS) Reception/Scheduling & 13.00 \\
\hline (W) Waiting & 9.75 \\
\hline (V) Vital & 7.80 \\
\hline (Wo) Workrooms & 7.09 \\
\hline (C) Consult Room & 6.50 \\
\hline (Ptt) Patient Toilet & 5.57 \\
\hline (CE) Clean/Med \& Equip Room & 5.57 \\
\hline (So) Soiled Room & 5.57 \\
\hline (L) Lobby & 5.20 \\
\hline (Sc) Social Support Office & 4.59 \\
\hline (Pt) Public Toilet & 3.00 \\
\hline
\end{tabular}

\begin{tabular}{|l|r|}
\hline Space from CD Floorplan & Integration \\
\hline c3 & 12.05 \\
\hline c2 & 11.00 \\
\hline c1 & 9.37 \\
\hline c5 & 7.23 \\
\hline (W) Waiting & 7.23 \\
\hline c6 & 6.84 \\
\hline c7 & 6.84 \\
\hline c4 & 6.33 \\
\hline c9 & 5.88 \\
\hline (V) Vital & 5.88 \\
\hline (Sc) Social Support Office & 5.88 \\
\hline (C) Consult Room & 5.88 \\
\hline (Wo) Workrooms & 5.62 \\
\hline (ExP) Exam/Procedure Rooms & 5.62 \\
\hline (Ptt) Patient Toilet & 5.16 \\
\hline (Ex) Exam Rooms & 5.16 \\
\hline c8 & 4.69 \\
\hline (L) Lobby & 4.69 \\
\hline (RS) Reception/Scheduling & 3.89 \\
\hline (CE) Clean/Med \& Equip Room & 3.69 \\
\hline (N) Nurse Sation & 5 \\
\hline (So) Soiled Room & \\
\hline (Pt) Public Toilet & \\
\hline
\end{tabular}


The four highest integrated spaces from the $\mathrm{CD}$ floorplan were the circulation spaces. This finding demonstrates the important role of circulation spaces in this clinical setting, and that there was a gap translating process flow into circulation spaces, and ultimately to floorplan layout. Some of those highly integrated circulation spaces were connected to functional spaces with the integration values that fell in the middle of the list such as vital room, exam rooms, and workrooms. In a glimpse, if the circulation spaces from the CD floorplan list are hidden, this observation list seems not to far from the one that was established for the process flow method (with the exception of exam rooms).

The integration value for nurse station drawn on the $\mathrm{CD}$ floorplan was low when compared to the one from the proposed method. Nurse station, which was designed with a counter, had its unique configuration, wherein the area was physically accessed from its sides (i.e., c6 and c7), but had additional visual access from c1. Even though the nurse station was visually connected to a corridor space with high integration value (i.e., $\mathrm{c} 1$ ), the value was relatively low when it was compared to exam rooms and workrooms, which were also connected to high integration corridor spaces (i.e., c1 and c2). We suggest for a more reliable method to develop in order to analyze this comparison for future studies.

In association with the first research objective, it seemed logical to develop space connections in JPG based on process flow. For example, one alternative of the patient flows was as follows: check-in from the lobby, register at the reception/ scheduling area, wait at the waiting area, call into the vital room, see the physician at the exam room, consult at the consult room, schedule next visit at the reception/scheduling area, and exit from the lobby. Thus, it was logical to see a fairly unobstructed connections of the following spaces in the JPG: lobby, to reception/scheduling, to waiting area, to vital room, to exam room, to consult room, to reception/scheduling, and finally to lobby (Figure 15). The JPG from the CD floorplan demonstrated a fairly unobstructed connections from the lobby, to waiting, to $c 3$, to vital, to $c 3$, to $c 1$, to exam rooms (Figure 16). However, from the exam rooms to the consult room, patients would have to walk five step depths, through several corridor spaces (i.e., c1, c3, c4, and c8). According to Haq and Zimring (2003), integration that are built from two step depths, named integration-3, had stronger correlation with wayfinding abilities. Thus, the exam - consult rooms' configuration could potentially delay patient flow from exam room to consult room, which could then impact care delivery process. Even though the $\mathrm{CD}$ floorplan layout was not only developed based on process flow, comparing the JPG analysis with the one from space adjacency analysis was beneficial in checking whether or not the development of the layout throughout building design process was still aligned with the project PR and, as in this particular example, patient flow and evidence based design on wayfinding in healthcare setting.

Further research ideas that could be developed based from these findings are:

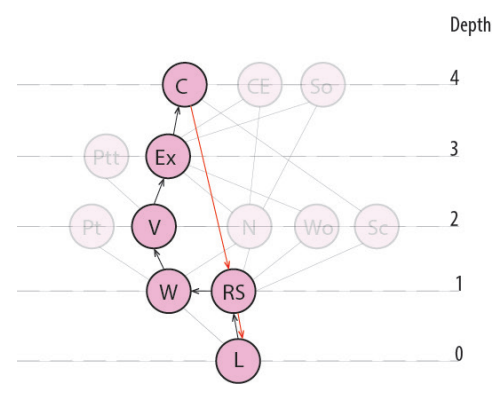

Figure 15: Process Flow JPG for Patient Flow.

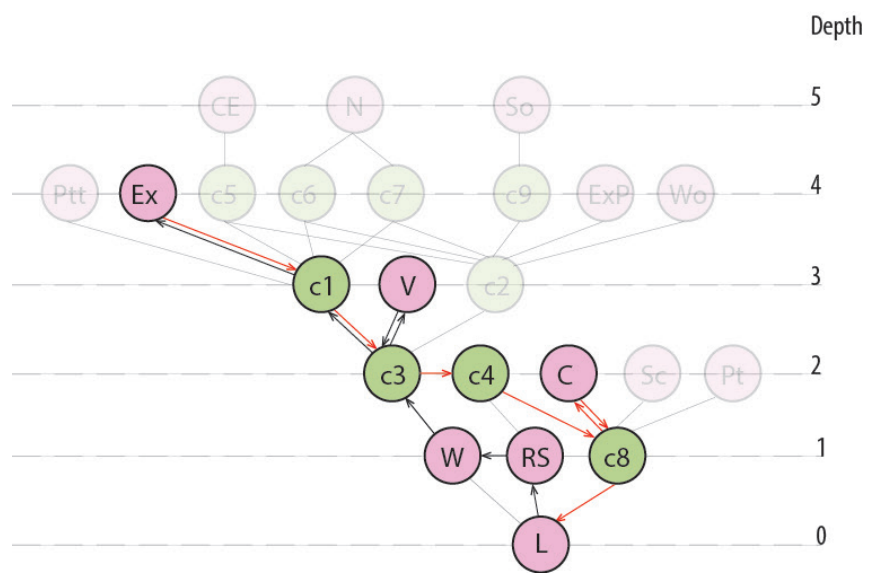

Figure 16: CD Floorplan JPG for Patient Flow.

- There is a need for method(s) that can incorporate circulation spaces in the space adjacency analysis or building programming phase or incorporated circulation spaces in the next design stages (e.g, schematic design, design development, and construction document) based on process flow information.

- If circulation spaces are not incorporated in the building programming phase, then appropriate method(s) to analyze the drawn floorplan with its high integration circulation spaces needs to be developed.

- Many studies that were related to nurses' movements and their locations to interact were investigated with space syntax visibility analysis technique into existing or drawn floorplan(s) (Haq \& Luo, 2012). However, is there any method to capture the uniqueness of nurse station's integration that can be implemented in the building programming phase, when a floorplan has not been drawn?

- There are many factors that could influence the output of space adjacency analysis such as noise, privacy, daylight, and many more. This study only explored process flow as one of the essential factors for space adjacency. Other important factors can be added and developed in future studies.

\section{Discussion}


Healthcare facilities are known to be complicated building types because they must provide the complex functions of healthcare services. Many specialized consultants from different fields play an important role in the planning and design of these facilities. This study proposes a computational method for integrating information and techniques from the fields of healthcare systems and of architecture into the building programming process. The basic approach for these integrations was abstracting the complexity of information among process flow, space syntax, and space adjacency fields into programmatic space and its connections. This demonstrated a more structured space adjacency PR process and the ability to integrate healthcare system and configurational analysis information in the building programming phase. The validity and reliability of this initial concept remains to be seen. The major finding, after comparing the analysis with one from the CD floorplan, was the limitation for integrating and analyzing circulation spaces, which can be addressed in future studies.

The comparison process as validity assessment offered additional consideration for checking the layout development throughout building design process towards design goals, performance requirements, and evidence based design. Jeong and Ban (2011) offered this computational ability for subtracting layout data from IFC, and then evaluating its designs and alterations using space syntax in order to reach desired design solution. If the data model structures for generating space adjacency and evaluating design solutionsare the same, then the programmers' and designers' involvement in the checking process can occured in a fairly seamless manner. The checking and alterations processes could also then potentially be used to alter the process flow and predict the care delivery outcome. Additional experimentations and developments of the computational, data and process models that focus on integration of information from different fields and programmers' creative and analytical way of thinking, will ultimately offer formalization of computational method in building programing process.

\section{Acknowledgments}

The research was part of a project funded by the Digital Building Laboratory Research Project (http://www.dbl. gatech.edu) at Georgia Tech, Atlanta, Georgia, USA. We wish to thank Diana Davis and Josh Emig from Perkins + Will (http://perkinswill.com/) for providing case study and data.

\section{Reference}

Agency for Healthcare Research Quality. (2014, February). Effective health care program: Stakeholder guide 2014 (AHRQ Publication No. 14-EHC010-EF). Retrieved from: http://www. ahrq.gov/sites/default/files/wysiwyg/research/findings/ evidence-based-reports/stakeholderguide/stakeholdr.pdf Arnolds, I., Nickel, S., Shashaani, S., \& Wernz, C. (2012). Using simulation in hospital layout planning. Proceedings of WSC 2012. Berlin, Germany; December 9-12. Retrieved May 20, 2015 from http://citeseerx.ist.psu.edu/viewdoc/ download?doi=10.1.1.416.912\&rep=rep1\&type=pdf

Bafna, S. (2001). Geometric intuitions of genotypes. Proceedings of the Third International Symposium on Space Syntax. Atlanta, 20.1-20.16.

Duerk, D. P. (1993). Architectural programming: Information management for design. New York: Van Nostrand Reinhold. Foulds, L. R. (1983). Techniques for facilities layout: Deciding which pairs of activities should be adjacent. Management Science, 29(12): 1414-1426.

Hall, R., Belson, D., Murali, P., \& Dessouky, M. (2006). Modeling patient flows through healthcare system. In Hall, R. (Eds.), Patient flow: reducing delay in healthcare delivery (1-44). Springer Science \& Business Media.

Haraden, C., \& Resar, R. (2004). Patient Flow in Hospitals: Understanding and Controlling It Better. Frontiers of Health Services Management, 20(4), 3-15.

Haq, S. (2001). Complex architectural settings: An investigation of spatial and cognitive variables through wayfinding behavior. PhD Dissertation, Georgia Institute of Technology, Atlanta.

Haq, S., \& Luo, Y. (2012). Space Syntax in healthcare facilities research: A review. Health Environments Research \& Design Journal, 5(4), 98-117.

Haq, S., \& Zimring, C. (2003). Just down the road a piece: The development of topological knowledge of building layouts. Environment and Behavior, 35(1), 132-160.

Hillier, B. \& Hanson, J. (1984). The social logic of space. New York: Cambridge University Press.

Jacobson, S. H., Hall, S. N., \& Swisher, J. R. (2006). Discreteevent simulation of health care systems. In Hall, R. (Eds.), Patient flow: reducing delay in healthcare delivery (1-44). Springer Science \& Business Media.

Jeong, S. K., \& Ban, Y. U. (2011). Computational algorithms to evaluate design solutions using Space Syntax. ComputerAided Design, 43(6), 664-676. http://doi.org/10.1016/j. cad.2011.02.011

Kim, T. W., Kim, Y., Cha, S. H., \& Fischer, M. (2015). Automated updating of space design requirements connecting user activities and space types. Automation in Construction, 50, 102-110. http://doi.org/10.1016/j.autcon.2014.12.010

Kumar, S. (2011). Modeling hospital surgical delivery process design using system simulation: Optimizing patient flow and bed capacity as an illustration, Technology and Health Care, 19: 1-20.

Lorenz, W. E., Bicher, M., \& Wurzer, G. X. (2015). Adjacency in hospital planning. IFAC-PapersOnLine, 48(1), 862-867. http:// doi.org/10.1016/j.ifacol.2015.05.118

McLaughlin, C. P., \& Kaluzny, A. D. (2006). Continuous Quality Improvement in Health Care: Theory, Implementations, and Applications. Jones \& Bartlett Learning.

medicaid.gov (2015), Affordable Care Act. Retrieved Jan, 2015 from http://medicaid.gov/affordablecareact/affordable-careact.html 
Michalek, J. J., Choudhary, R., \& Papalambros, P. (2002). Architectural Layout Design Optimization. Engineering Optimization, 34(5): 461-484.

Nourian, P., Rezvani, S., \& Sariyildiz, S. (2013). Designing with Space Syntax: A configurational approach to architectural layout, proposing a computational methodology. In Computation and Performance - Proceedings of the 31st eCAADe Conference.Delft, The Netherlands: Delft University of Technology,1: 357-365.

Ostwald, M. J. (2011). The mathematics of spatial configuration: Revisiting, revising and critiquing justified plan graph theory. Nexus Network Journal, 13(2): 445-470.

Peña, W. M., \& Parshall, S. A. (2012). Problem seeking: An architectural programming primer. New York: Wiley.

Peponis, J., Zimring, C., \& Scanlon, M. (1996). New design technologies using computer technology to improve design quality. Journal of Healthcare Design, VIII, 109-119.

Potisek, N. M., Malone, R. M., Shilliday, B. B., Ives, T. J., Chelminski, P. R., DeWalt, D. A., \& Pignone, M. P. (2007). Use of patient flow analysis to improve patient visit efficiency by decreasing wait time in a primary care-based disease management programs for anticoagulation and chronic pain: a quality improvement study. BMC Health Services Research, 7(1), 8. http://doi.org/10.1186/1472-6963-7-8

White, E. T. (1986). Space adjacency analysis: Diagramming information for architectural design. Architectural Media Ltd.

Wurzer, G. (2013). In-process agent simulation for early stages of hospital planning. Mathematical and Computer Modelling of Dynamical Systems, 19(4), 331-343. http://doi.org/10.1080/ 13873954.2012.761638 